
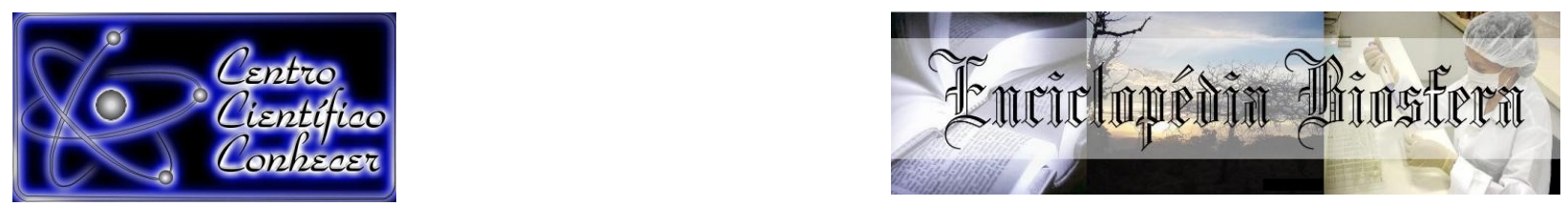

\title{
REFLEXÕES SOBRE O DIAGNÓSTICO PRECOCE DO CÂNCER DE MAMA: UM ESTUDO DE CASO ACERCA DA FAIXA ETÁRIA ALVO DAS AÇÕES DE RASTREIO
}

Ana Luiza de Souza Francioli ${ }^{1}$, Alan Deniver Chimenes Barbosa ${ }^{2}$, Mirian Ueda Yamaguchi ${ }^{3}$, Tânia Maria Gomes da Silva ${ }^{4}$, Marcelo Picinin Bernuci ${ }^{5}$

Acadêmica do Curso de Graduação em Medicina do Centro Universitário de Maringá UNICESUMAR - Maringá-PR, Brasil. Bolsista PROBIC

${ }^{2}$ Acadêmico do Curso de Graduação em Medicina do Centro Universitário de Maringá UNICESUMAR - Maringá-PR, Brasil. Bolsista PROBIC

${ }^{3}$ Docente do Programa de Pós-Graduação em Promoção da Saúde do Centro Universitário de Maringá - UNICESUMAR - Maringá-PR, Brasil. Pesquisadora, Bolsista Produtividade em

Pesquisa do Instituto Cesumar de Ciência,Tecnologia e Inovação - ICETI

${ }^{4}$ Docente do Programa de Pós-Graduação em Promoção da Saúde do Centro Universitário de Maringá - UNICESUMAR - Maringá-PR, Brasil. Pesquisadora, Bolsista Produtividade em

Pesquisa do Instituto Cesumar de Ciência,Tecnologia e Inovação - ICETI. tania.gomes@unicesumar.edu.br

${ }^{5}$ Docente do Programa de Pós-Graduação em Promoção da Saúde do Centro Universitário de Maringá - UNICESUMAR - Maringá-PR, Brasil. Pesquisador, Bolsista Produtividade em

Pesquisa do Instituto Cesumar de Ciência,Tecnologia e Inovação - ICETI

Recebido em: 06/04/2018 - Aprovado em: 10/06/2018 - Publicado em: 20/06/2018 DOI: 10.18677/EnciBio_2018A105

Trata-se de um estudo retrospectivo de dados secundários obtidos no Sistema de Informação de Câncer (SISCAN) a fim de avaliar as ações de detecção precoce do câncer de mama no Município de Maringá, Paraná. Os dados referem-se a informações relativas à quantidade de mamografias e biópsias realizadas, das mulheres em seguimento, diagnosticadas ou não com câncer de mama entre os anos de 2014 e 2015. Foram realizadas 8.268 mamografias, sendo que $78,66 \%$ destas em mulheres na faixa etária de $50-69$ anos e $21,33 \%$ fora desta faixa. Foram realizadas 53 biópsias, sendo que $54,71 \%$ na população alvo e $45,28 \%$ fora desta. Os resultados de biopsia foram $79,31 \%$ positivas e $20,65 \%$ negativas na faixa etária da população alvo e $75 \%$ positivas e $25 \%$ negativas fora da faixa etária alvo. Entre as pacientes com histologia positiva, a maior frequência foi observada para a classificação 5 no sistema BI-RADS, enquanto que para aquelas que apresentaram resultado histológico negativo, a metade obteve classificação 3 no BI-RADS. Conclui-se que o excesso de zelo do serviço avaliado em expor um número considerável de mulheres fora da faixa etária preconizada à realização de mamografia e exame histopatológico garantiu a detecção precoce das lesões malignas, reforçando o debate sobre uma reavaliação das regras de cobertura dos exames preventivos.

PALAVRAS-CHAVE: câncer de mama, mamografia, prevenção 


\title{
REFLECTIONS ON EARLY CANCER DIAGNOSIS OF BREAST CANCER: A CASE STUDY ABOUT THE AGE GROUP TARGETED OF SCREENING ACTIONS
}

\begin{abstract}
This is a retrospective study of secondary data obtained from the Cancer Information System (SISCAN) in order to evaluate the early detection of breast cancer in the city of Maringá, Paraná. The data refer to information regarding the number of mammograms and biopsies performed in women in the follow-up, diagnosed or not with breast cancer between the years 2014 and 2015. There were 8,268 mammograms, of which $78.66 \%$ were women in the age group of $50-69$ years and $21.33 \%$ outside this range. 53 biopsies were performed, with $54.71 \%$ in the target population and $45.28 \%$ outside this range. The biopsy results were $79.31 \%$ positive and $20.65 \%$ negative in the age group of the target population and $75 \%$ positive and $25 \%$ negative outside the target age group. Among the patients with positive histology, the highest frequency was observed for classification 5 in the BI-RADS system, while for those who presented negative histological results, half had a BIRADS classification 3. It was concluded that the overzealousness of the service evaluated in exposing a considerable number of women outside the age range recommended for mammography and histopathological examination ensured the early detection of malignant lesions, reinforcing the debate about a reassessment of the coverage rules of the exams preventive measures.
\end{abstract}

KEYWORDS: breast cancer, mammography, prevention

\section{INTRODUÇÃO}

Mortes relacionadas a neoplasias malignas têm sido cada vez mais frequentes na população mundial (TORRE et al., 2015). Dentre os diferentes tipos, destaca-se o câncer de mama, o segundo tipo mais comum de câncer no mundo (LUQUE et al., 2018; MACHADO et al., 2017). Estimativas informam que, em 2020, haverá 1,7 milhão de novos casos de câncer de mama nos países em desenvolvimento (RIVERA-FRANCO ; LEON-RODRIGUEZ, 2018). Desde a segunda metade do século $X X$, mudanças no cotidiano das mulheres, ao menos nos países ocidentais, como maior ingresso no mercado de trabalho, aumento no consumo de álcool e cigarro, obesidade, uso de anticoncepcionais e maternidade tardia, associadas a fatores genéticos e ambientais, têm contribuído para progressão nas taxas de câncer de mama (RODRIGUES et al., 2015; OHL et al., 2016).

Embora a incidência e mortalidade reduzam à medida que práticas de detecção precoce são adotadas pela população, não se tem observado redução expressiva nos índices de incidência e mortalidade por essa neoplasia no Brasil (CARIOLI et al., 2018). De fato, o câncer de mama representa a primeira causa de morte por câncer na população feminina brasileira, e já é considerada uma questão de saúde pública (INCA, 2017; COUTO et al., 2017). Além disso, reconhece-se que as taxas de sobrevivência de cinco anos para mulheres que apresentaram câncer de mama são piores para países como o Brasil $(58,4 \%)$, Índia (52\%), Argélia $(38,8 \%)$ e Gâmbia (12\%), em comparação com os Estados Unidos (83,9\%), Suécia $(81,6 \%)$ e Austrália (80,7\%) (RODRIGUES et al., 2015). Estudos epidemiológicos entendem essa desigualdade em parte devido a fatores socioeconômicos, que implicam em acesso desigual à qualidade de saúde e reduzem a taxa de acesso à mamografia, 
mas não desconsideram os fatores genéticos que fogem ao controle das mulheres, afirmam os autores citados acima.

De fato, a mamografia de rastreio regular para detecção precoce do câncer de mama tem sido amplamente recomendada e é apoiada por evidências sólidas, haja vista que a maioria dos ensaios clínicos randomizados de base populacional relataram menor mortalidade entre mulheres submetidas a mamografia de rastreio (PACE ; KEATING, 2014; OEFFINGER et al., 2015; TABAR et al., 2015; NIELL et al., 2017; PARK et al., 2017). Países que investiram fortemente em ações de detecção precoce, como Canadá e Austrália, conseguiram grandes avanços na redução da mortalidade por este tipo de câncer. Apenas entre os anos de 2002 a 2012 os resultados apontam para reduções de mais de $20 \%$ na taxa de mortalidade nestes países, enquanto que no Brasil esta taxa não supera 6\% (CARIOLI et al., 2018). Falha no seguimento das recomendações do Mistério da Saúde para a detecção precoce pelos profissionais envolvidos tem sido sugerida como um dos motivos da baixa expressividade das ações de controle desta malignidade no Brasil (TOMAZELLI et al., 2017), o que suscita a necessidade de constante avaliação da atuação dos serviços de saúde no controle dessa neoplasia.

Neste tocante, a falta de monitoramento dos resultados das mamografias e de ações corretivas para melhoria de qualidade das mesmas pode, portanto, promover uma redução da acurácia do sistema preventivo, o que aumentará a probabilidade de mulheres com câncer de mama não serem identificadas nos exames de rastreamento (falso-negativos) e de mulheres sem câncer de mama receber um resultado falso-positivo. Assim, o presente estudo objetivou caracterizar as ações de diagnóstico precoce do câncer de mama a fim de apontar melhoras para o sistema e subsidiar novos debates sobre as diretrizes de rastreio do câncer de mama no país.

\section{MATERIAL E MÉTODOS}

Tratou-se de estudo descritivo de delineamento retrospectivo de dados secundários obtidos no Sistema de Informação de Câncer (SISCAN) (BRASIL, 2013), instalado em computadores da coordenação da $15^{a}$ Regional de detecção precoce de câncer de mama do Município de Maringá, Paraná. Os dados referem-se a informações relativas à quantidade de mamografias e biópsias realizadas em mulheres diagnosticadas ou não com câncer de mama no Município, entre os anos de 2014 e 2015. De acordo com o último recenseamento, a população feminina maringaense entre 40 a 69 anos era de 48.421 mulheres (MATOS et al., 2011).

Após a tabulação dos dados em planilhas do Excel, calcularam-se as seguintes taxas: a) proporção de mamografias de rastreamento na faixa etária preconizada, dada por número de mamografias de rastreamento em mulheres de 50 a 69 anos, residentes em dado local e período x $100 / \mathrm{N}^{\circ}$ total de mamografias de rastreamento no respectivo local e período; b) percentual de exames histopatológicos liberados em até 30 dias, dada por número de exames histopatológicos (por biópsias) realizados em até 30 dias x 100 /Total de exames histopatológicos (por biópsias) realizados.

$\mathrm{O}$ valor preditivo positivo (VPP) foi também determinado e referiu-se à proporção de indivíduos que apresentaram a doença a partir de testagem específica, dentre aqueles que obtiveram resultado positivo. Neste caso, a proporção é relativa ao número de biópsias positivas das pacientes para as quais a mesma foi indicada através da mamografia. A prevalência estimada (PE) também foi determinada e 
referiu-se à proporção de pacientes que também realizaram a biópsia dentre aquelas que realizaram a mamografia.

Objetivando verificar a associação entre a classificação no sistema BI-RADS e o resultado histológico das pacientes pesquisadas, aplicou-se o teste exato de Fisher. As análises estatísticas foram obtidas com o auxílio do software ambiente estatístico R.

\section{RESULTADOS E DISCUSSÃO}

Os dados referentes à proporção de mamografias realizadas dentro e fora da faixa etária estabelecida pelo Ministério da Saúde estão apresentados na Tabela 1. Nota-se que, no período de 2014 a 2015, em Maringá, foram realizadas 8.268 mamografias de rastreamento, sendo que $78,66 \%$ (6.504) em mulheres na faixa etária de 50-69 anos e 21,33\% (1.764) fora desta faixa.

TABELA 1 Proporção de mamografias realizadas dentro e fora da faixa etária estabelecida pelo Ministério da Saúde no período de 2014 a 2015 na região de Maringá-Pr

\begin{tabular}{c|ccc} 
& $\begin{array}{c}\text { FAIXA ETARIA DE } \\
\mathbf{5 0 - 6 9 A}\end{array}$ & $\begin{array}{c}\text { FORA DA FAIXA } \\
\text { ETÁRIA ANTERIOR } \\
(<50 \text { E >69A) }\end{array}$ & TOTAL \\
$\begin{array}{c}\text { MAMOGRAFIAS DE } \\
\text { RASTREAMENTO }\end{array}$ & 6504 & 1764 & 8268 \\
$\%$ & 78,66 & 21,33 & 100
\end{tabular}

\section{CARACTERIZAÇÃO DAS AÇÕES DE DIAGNÓSTICO HISTOPATOLÕGICO}

Os dados referentes à relação de biópsias positivas e negativas de acordo com a idade estão apresentados na Tabela 2. No período investigado, foram realizadas em Maringá 53 biópsias, sendo que $54,71 \%$ (29) na população alvo e $45,28 \%$ (24) fora desta. Os resultados de biopsia foram $79,31 \%$ (23) positivas e $20,65 \%$ (6) negativas na faixa etária da população alvo e $75 \%$ (18) positivas e $25 \%$ (6) negativas fora da faixa etária alvo (Tabela 2).

TABELA 2 Relação de biópsias positivas e negativas de acordo com a idade realizadas no período de 2014 a 2015 na região de Maringá-Pr 


\begin{tabular}{|c|c|c|c|}
\hline & $\begin{array}{c}\text { FAIXA ETÁRIA DE } \\
50-69 A\end{array}$ & $\begin{array}{c}\text { FORA DA FAIXA } \\
\text { ETÁRIA }(<50 \mathrm{E} \\
>69 A)\end{array}$ & TOTAL \\
\hline $\begin{array}{c}\text { BIÓPSIAS } \\
\text { POSITIVAS PARA } \\
\text { CA }\end{array}$ & 23 & te & 41 \\
\hline $\begin{array}{c}\text { BIÓPSIAS } \\
\text { NEGATIVAS PARA } \\
\text { CA }\end{array}$ & 6 & 6 & 12 \\
\hline $\begin{array}{c}\text { TOTAL DE } \\
\text { BIÓPSIAS } \\
\text { POSITIVAS E } \\
\text { NEGATIVAS PARA } \\
\text { CA }\end{array}$ & 29 & 24 & 53 \\
\hline
\end{tabular}

\section{CARACTERIZAÇÃO DAS AÇÕES DE CONFIRMAÇÃO DIAGNÓSTICA}

A Tabela 3 apresenta os dados referentes ao valor preditivo positivo (VPP) e prevalência estimada (PE) do câncer de mama pela mamografia, de acordo com a faixa etária. Observou-se que, as pacientes entre 50 e 69 anos apresentaram um VPP maior que aquelas fora da faixa etária, o que significa que, de cada 100 biópsias requisitadas por meio da mamografia, cerca de 80 (VPP $=0,79)$ biópsias apresentam resultado positivo. No geral, esta confirmação pela biópsia ocorreu em $77 \%$ dos casos.

TABELA 3 Valor preditivo positivo e prevalência estimada do câncer de mama pela mamografia, de acordo com a faixa etária realizada em pacientes no período de 2014 a 2015 na região de Maringá-Pr

\begin{tabular}{l|ccccc}
\hline & Mamografias & $\begin{array}{c}\text { Biópsias } \\
(+)\end{array}$ & $\begin{array}{c}\text { Biópsias } \\
(-)\end{array}$ & VPP & PE \\
\hline Faixa etária & & & & & 0,00 \\
$\quad \begin{array}{l}50|-| 69 \text { anos } \\
\quad 50 \mathrm{e}>69\end{array}$ & 6504 & 23 & 6 & 0,79 & $\begin{array}{l}4 \\
0,01\end{array}$ \\
anos & 1764 & 18 & 6 & 0,75 & 4 \\
\hline Total & 8268 & 41 & 12 & 0,77 & 6,00 \\
\hline
\end{tabular}

Nota-se na Figura 1 que em média, a idade das pacientes pesquisadas foi superior para aquelas que apresentaram histologia positiva em comparação com o resultado negativo (59 e 51 anos, respectivamente). Em geral, sem considerar o resultado histológico, a média e a mediana da idade coincidem em 57 anos. 


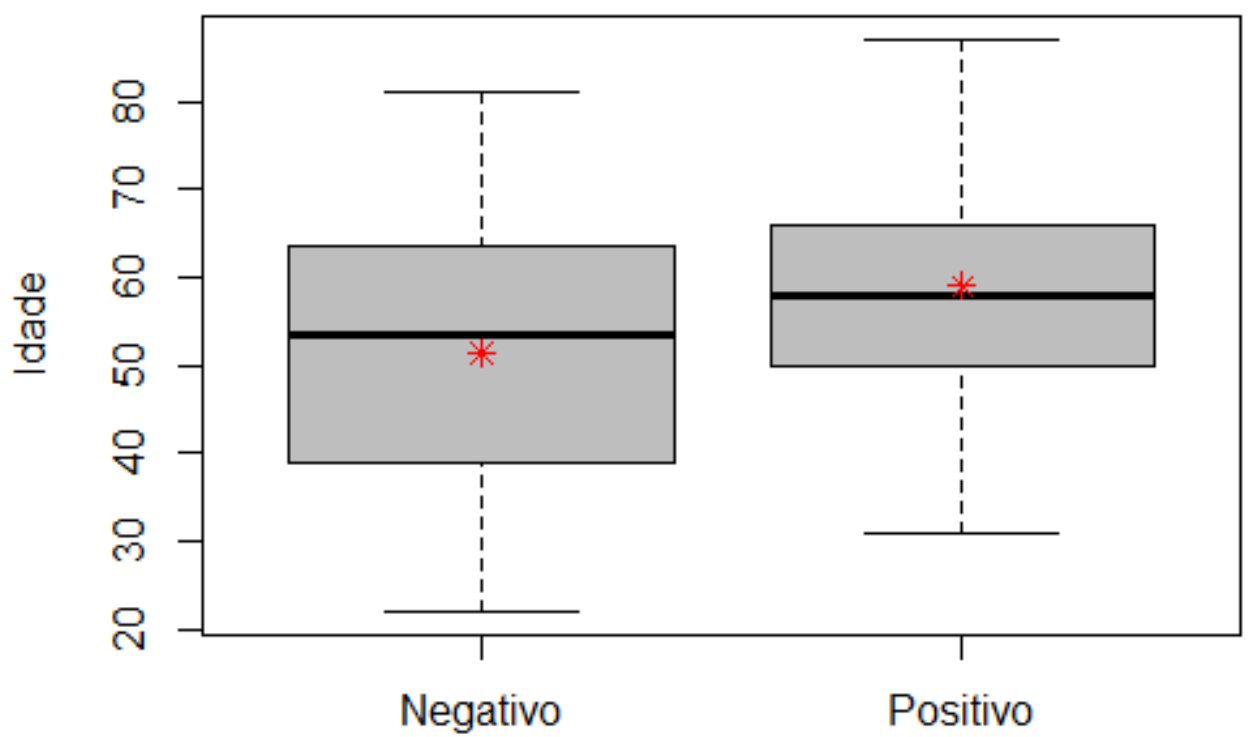

FIGURA 1 Distribuição da idade (em anos) das pesquisadas de acordo com o resultado das biópsias realizadas nas pacientes no período de 2014 a 2015 na região de Maringá-Pr

Os dados referentes à associação entre a classificação BI-RADS e a histologia das pacientes estão apresentados na Tabela 4. Verificou-se que, entre as pacientes com histologia positiva, a maior frequência foi observada para a classificação 5 no sistema BI-RADS, enquanto que para aquelas que apresentaram resultado histológico negativo, a metade obteve classificação 3 no BI-RADS. Como apresentado, existem indícios amostrais de que a classificação BI-RADS está associada com o resultado histológico, ao nível de $5 \%$ de significância (valor $p$ de 0,0266).

TABELA 4 Distribuição de frequências e resultado do teste exato de Fisher para a associação entre a classificação BI-RADS e o resultado das biópsias realizadas nas pacientes no período de 2014 a 2015 na região de Maringá-Pr

\begin{tabular}{c|cc|c}
\hline BI-RADS & \multicolumn{2}{|c|}{$\begin{array}{c}\text { Histologia } \\
\text { Negativo Positivo }\end{array}$} & $\begin{array}{c}\text { Teste exato de } \\
\text { Fisher } \\
\text { Valor } \mathbf{p}\end{array}$ \\
\hline 0 & 1 & 0 & \\
1 & 1 & 3 & \\
2 & 1 & 5 & 0,0266 \\
3 & 4 & 6 & \\
4 & 1 & 9 & \\
5 & 0 & 13 & \\
\hline
\end{tabular}

No Brasil, existem recomendações quanto à população-alvo e periodicidade dos exames: mulheres na faixa etária de 40 a 49 anos devem realizar exame clínico das mamas (ECM) anualmente $e$, em caso de alteração, deve-se realizar mamografia. Em mulheres de 50 a 69 anos, o ECM deve ser anual e a mamografia 
ocorrer de dois em dois anos. Já aquelas com 35 anos ou mais, com risco elevado, devem realizar tanto o ECM quanto a mamografia todos os anos (MORAES et al., 2016). Considerando-se o último informativo de detecção precoce do câncer de mama, realizado em 2013, a proporção de mamografias de rastreamento na faixa etária alvo deve ser de $50 \%$ (INCA, 2015). Na avaliação realizada no presente estudo, a proporção de mamografias de rastreamento na faixa etária alvo no Município de Maringá apresentou índices muito acima da média preconizada.

O estudo mostrou que mais de $20 \%$ das mulheres fora da faixa etária realizaram a mamografia. É provável que estas tenham sido encaminhadas para confirmação diagnóstica após suspeita no exame clínico das mamas ou terem sido indicadas devido ao risco elevado. Como estas informações não estão presentes no sistema de informação, a caracterização da dinâmica do processo de diagnóstico precoce fica prejudicada, e reafirma a necessidade de reajustes nos sistemas de informação.

De qualquer forma, sem o conhecimento da história da paciente no sistema, a conclusão que se pode chegar para o Município de Maringá é dúbia. Pode-se entender que muitas mulheres são expostas ao exame mamográfico sem necessidade, ou que de fato essa população está sob um risco elevado e merece investigação mais cuidadosa, destacando assim, favoravelmente, a atuação do serviço no controle da malignidade.

O exame de mamografia é realizado a fim de investigar lesões palpáveis e detectar lesões não palpáveis de mama. A conduta nas lesões identificadas segue a proposta do Breast Imaging Reportingand Data ${ }^{\circledR}$ System (BI-RADS). Nas lesões Categoria 4 e 5 (BI-RADS) está indicado estudo histopatológico. Os critérios histopalógicos para a avaliação das lesões mamárias devem ser categorizados a princípio como: padrão citológico benigno ou negativo para malignidade (INCA, 2015). Portanto, avaliar os resultados do estudo histopatológico de acordo com as faixas etárias preconizadas para a mamografia pode indicar se de fato o excesso de zelo no rastreio mamográfico apresentado no serviço do Município de Maringá é coerente ou não com o objetivo final do programa de controle da doença, ou seja, diagnosticar precocemente as lesões malignas.

Com isto, evidenciou-se a controvérsia em relação à faixa etária recomendada pelo Ministério da Saúde para realização da mamografia de rastreio no CA de mama, uma vez que existe um número significativo de diagnóstico de câncer fora da faixa etária (URBAN et al., 2012). Isso reforça a ideia de que tanto a indicação da realização da biopsia em mulheres fora da faixa etária recomendada pela suspeita no EC quanto pela indicação de alto risco são relevantes para o diagnóstico precoce, e, portanto, o aparente excesso de zelo, pelo menos no presente caso, é necessário. E mostra, mais uma vez que o serviço de diagnóstico precoce prestado pelo Município tem sido eficiente e reacende as discussões acerca das recomendações do Ministério da Saúde.

Nota-se também que em Maringá, o tempo de espera entre a coleta do exame histopatológico de mama e o resultado foi menor do que 30 dias (dados não mostrados), sugerindo que o serviço de detecção precoce de câncer de mama tem cumprido com o intervalo de tempo preconizado pelo Ministério da Saúde entre a coleta do exame histopatológico e o resultado, destacando ainda mais a atuação positiva de Maringá no controle deste tipo de câncer. 
Não se descarta que o número de mulheres em Maringá que realizou exame mamográfico fora da faixa etária preconizada pode ter sido em razão de indicação prescrita pós-exame clínico com resultado indefinido, ou por encaminhamento médico dispensável. A incompletude dos prontuários analisados não permitiu uma resposta conclusiva, mas, admitindo-se a segunda hipótese, pode se pensar que as pacientes estiveram expostas desnecessariamente aos impactos psicológicos de achados falso-positivos, com dispêndio de recursos que o setor de saúde poderia alocar, por exemplo, na melhoria da qualidade das mamografias. No entanto, muitas suspeitas indicadas na mamografia em mulheres fora da faixa de rastreio foram confirmadas no exame histopatológico, sugerindo de fato a relevância de mais debates sobre a possibilidade de uma reavaliação do esquema de rastreio adotado no país.

Não se pode ignorar, contudo, os estudos que vão de encontro a esta sugestão. $\mathrm{O}$ número de mortes evitadas por mamografias de rastreamento em mulheres com idade entre 40 e 49 anos é menor do que em mulheres mais velhas e o número de resultados falso-positivos e biópsias desnecessárias é também maior havendo pouca efetividade na morbimortalidade de populações reais (SIU, 2016). Assim, é preciso evitar a supervalorização do rastreamento mamográfico e apresentar os possíveis riscos (SILVA et al, 2014).

Diante de tantas controvérsias e debates acerca da dinâmica de rastreio das lesões malignas de câncer de mama no país, o presente estudo contribui evidenciando as ações de controle do câncer de mama no Município de Maringá com a retomada das discussões dos excessos de cuidados em saúde, descritos em 1999, pelo médico belga Marc Jamoulle, como Prevenção Quaternária e que é rediscutido na atualidade tanto pela comunidade científica, quanto médica (NORMAN ; TESSER, 2016; TESSER, 2017).

Santos (2013), por sua vez, embora admita que a incidência de CA de mama seja menor na fase anterior aos 50 anos, ressalta que há risco de tumores com crescimento mais rápido, razão pela qual sugere maior acuidade por parte dos profissionais da saúde. Sendo assim, há de se avaliar com muito cuidados tanto os benefícios quanto os malefícios do rastreio em populações mais jovens.

As mulheres também precisam estar atentas a um discurso de saber/poder que desde os anos 1990 tem permeado as discussões relacionadas ao câncer de mama. Campanhas como as do laço rosa, por exemplo, que buscam isentar o Estado das responsabilidades para com a doença/doente, colocam as mulheres como "empresárias" de seus corpos e únicas responsáveis pelo seu processo de saúde/adoecimento. Fatores ambientais, a feminização da pobreza, atrasos educacionais e a desigualdade de gênero nunca são colocados em evidência nas campanhas direcionadas às mulheres. A tônica do discurso "rosa" é neoliberal e não de confrontação; o que o torna um movimento bastante palatável às agências financiadoras nacionais e internacionais. Além disso, no grupo daquelas que se "cuidam" e "não se deixam" morrer enquadram, quase sempre, as brancas, heterossexuais e de classe média (VASQUEZ, 2017). Todas estas questões são relevantes, e merecem ser melhor discutidas antes de se definir um novo protocolo de rastreio.

\section{CONCLUSÃO}


De forma geral, evidenciou-se que, no Município de Maringá, o programa de detecção precoce do câncer de mama em mulheres na faixa etária indicada está sendo realizado de acordo com as diretrizes do INCA, porém mais de $20 \%$ das mamografias foram realizadas fora da população preconizada. Este excesso de zelo no rastreio pode tanto expor desnecessariamente as mulheres e gerar gastos que poderiam ser direcionados para outros aspectos do programa de controle do câncer de mama como também pode garantir maior precocidade no achado das lesões malignas em mulheres mais jovens. Considera-se, assim, a necessidade de mais pesquisas avaliativas dos riscos e benefícios do rastreamento de modo a promover saúde aliada à qualidade de vida das mulheres.

\section{REFERÊNCIAS}

BRASIL. Ministério da Saúde. Secretaria de Atenção à Saúde. Departamento de Atenção Básica. Controle dos cânceres do colo do útero e da mama. 2. ed. Brasília: MS; 2013. Disponível em: http://bvsms.saude.gov.br/bvs/publicacoes/controle_canceres_colo_utero_2013.pdf.

CARIOLI, G.; MALVEZZI, M.; RODRIGUEZ, T.; BERTUCCIO, P.; NEGRI E.; LA VECCHIA, C. Trends and predictions to 2020 in breast cancer mortality: Americas and Australasia. Breast. v. 37, p. 163-169, 2018. Disponível em: https://www.ncbi.nlm.nih.gov/pubmed/29246526.http://dx.

DOI:10.1590/S0102-311X2011000500007:0.1016/j.

COUTO, M. S. A.; GUERRA, M. R.; FIRME, V. A. C.; BUSTAMANTE-TEIXEIRA, M. T. Comportamento da mortalidade por câncer de mama nos municípios brasileiros e fatores associados. Revista Panamericana de Salud Pública, v. 41, p. 1-10, 2017. Disponível em: https://www.scielosp.org/pdf/rpsp/2017.v41/e168/pt. DOI:10.26633/rpsp.2017.168.

INCA - Instituto Nacional de câncer José Alencar Gomes da Silva . Diretrizes para a detecção precoce do câncer de mama no Brasil. Rio de Janeiro, 2015. Disponível em: http://www2.inca.gov.br/wps/wcm/connect/4da965804a4414659304d3504e7bf539/Di retrizes+Detec\%C3\%A7\%C3\%A3o+Precoce+Ca+Mama+2015.pdf?

MOD=AJPERES\&CACHEID=4da965804a4414659304d3504e7bf539

INCA - Instituto Nacional de câncer José Alencar Gomes da Silva Estimativa 2018 - Incidência de Câncer no Brasil. Rio de Janeiro, 2017. Disponível em: http://www1.inca.gov.br/inca/Arquivos/estimativa-2018.pdf

LUQUE, J. S.; LOGAN, A.; SOULEN, G.; ARMESON, K. E. Systematic Review of Mammography Screening Educational Interventions for Hispanic Women in the United States. Journal of Cancer Education. American Association for Cancer Education, $\quad$ v. $12, \quad 2018 . \quad$ Disponível em: https://www.ncbi.nlm.nih.gov/pubmed/29330754. DOl:10.1007/s13187-018-1321-0.

MACHADO, M. X.; SOARES, D. A.; OLIVEIRA, S. B. Significados do câncer de mama para mulheres no contexto do tratamento quimioterápico. Physis. Revista de 
Saúde Coletiva. ${ }^{* *}-{ }^{*}{ }^{*} v . \quad 27, \quad$ n. $3, \quad$ p.433-451, 2017. Disponível em: http://www.scielo.br/scielo.php?

pid=S010373312017000300433\&script=sci_abstract\&tIng=pt. DOI: 10.1590/s010373312017000300004.

MATOS, J. C; PELLOSO, S.M; CARVALHO, M. D. de B. Fatores associados à realização da prevenção secundária do câncer de mama no Município de Maringá, Paraná, Brasil. Cadernos de Saúde Pública. v. 27, n. 5, p. 888898, 2011. Disponível EM: <http://www.scielo.br/scielo.php? script=sci_arttext\&pid=S0102. DOI: 10.1590/S0102-311X2011000500007.

MORAES, D.C.; ALMEIDA, A.M.; FIGUEIREDO, E. N.; LOYOLA, E.A.C.; PANOBIANCO, M.S. Rastreamento oportunístico do câncer de mama desenvolvido por enfermeiros da Atenção Primária à Saúde. Revista da Escola de Enfermagem da USP. v. $50, \quad$ n. 1 , p.14-21, 2016. Disponível em: http://www.scielo.br/pdf/reeusp/v50n1/pt_0080-6234-reeusp-50-01-0014.pdf. DOI: 10.1590/S0080-623420160000100002.

NIELL, B..L.; WEINFURTNER, R. J.; ARLEO, E., K.; DRUKTEINIS, J. S. Screening for breast cancer. Radiolologic Clinics of North America. v. 55, n. 6, p.1145-1162, 2017. Disponível em: https://www.ncbi.nlm.nih.gov/pubmed/28991557. DOI 10.1016/j.rc.2017.06.004.

NORM, A. H., TESSER, C. D., Prevenção quaternária na atenção primária à saúde: uma necessidade do Sistema Único de Saúde. Cadernos de Saúde Pública. Rio de Janeiro, v. 25, n. 9, p. 2012-2020, 2009. Disponível em: http://www.scielo.br/pdf/csp/v25n9/15.pdf. DOI: 10.1590/S0102311X2009000900015.

OEFFINGER, K. C.; FONTHAM, E. T.; ETZIONI, R.; HERZIG, A.; MICHAELSON, J. S.; SHIH, Y; C.; et al.,; R. Breast Cancer Screening for Women at Average Risk: Guideline Update from the American Cancer Society. JAMA._V. 20, n. 15, p.1599-1614. 2015.2 Disponível em: https://www.ncbi.nlm.nih.gov/pmc/articles/PMC4831582/.

DOI: 10.1001/jama.2015.12783.

OHL, I. C. B.; OHL, R. I. B.; CHAVAGLIA, S. R. R.; GOLDMAN, R. E. Public actions for control of breast cancer in Brazil: integrative review. Revista Brasileira de Enfermagem. v. 69, n. 4, p. 793-803, $2016 . \quad$ Disponível em: <http://www.scielo.br/scielo.php?script=sci_arttext\&pid=S003471672016000400793\&Ing=en\&nrm=iso>.DOI: 10.1590/0034-7167.2016690424i. 
PACE, L.; KEATING, N. L. A systematic assessment of benefits and risks to guide breast cancer screening decisions. JAMA. v. 2, n. 13, p. 1327-1335, 2014. U. S National Library of Medicine. National Institute of Health. Disponível em: https://www.ncbi.nlm.nih.gov/pubmed/24691608. DOI: 10.1001/jama.2014.1398.

PARK, J.M.; BAE S.J.; YOON,C.; LEE, H.S.; LEE, H.W.; AHN, S.G.; LEE, S.A.; JEONG, J. Comparison of patients with small $(\leq 2 \mathrm{~cm})$ breast cancer according to adherence to breast screening program. PLos One. v. 2, n. 11, p. 110, 2017. Disponível em: 0186988.http://dx. DOI: 10.1371/journal.pone.0186988.

RIVERA-FRANCO, M. M.; LEON-RODRIGUEZ, E. Delays in Breast Cancer Detection and Treatment in Developing Countries. Breast Cancer: Basic and Clinical Research. v. 12, n. 8, p. 1-5, 2018. Disponível em: https://www.ncbi.nlm.nih.gov/pmc/articles/PMC5802601/pdf/10.1177_117822341775 2677.pdf. DOI: 10.1177/1178223417752677.

RODRIGUES, J. D.; CRUZ, M. S.; PAIXAO, A. N. Uma análise da prevenção do câncer de mama no Brasil. Ciência e saúde coletiva. v. 20, n. 10, p. 31633176, 2015. Disponível em: <http://www.scielo.br/scielo.php? script=sci_arttext\&pid=S141381232015001003163\&lng=en\&nrm=iso>.

DOI:10.1590/1413-812320152010.20822014.

SANTOS, J. A. Rastreamento do câncer de mama: as três luzes do semáforo. Revista Brasileira de Medicina. v. 8, n. 26, p. 11-15, 2013. Disponível em: https://rbmfc.org.br/rbmfc/article/view/677/535. DOI: 10.5712/rbmfc8.

SILVA, F. X. S.; KATZL, L.; SOUZA, A.S.R.; AMORIN, M. M.R. Mamografia em mulheres assintomáticas na faixa etária de 40 a 49 anos. Revista de Saúde Pública. v. 48, n. 6, p. 931-939, 2014. Disponível em: http://www.scielo.br/pdf/rsp/v48n6/pt_0034-8910-rsp-48-6-0931.pdf. DOI: 10.1590/S0034-8910.2014048005349.

SIU, A.L. Screening for breast câncer: U.S. Preventive Services Task Force Recommendation Statement. Ann International Medicine. v. 164, n. 4, p. 279296, 2016. Disponível em: http://annals.org/aim/fullarticle/2480757/screening-breastcancer-u-s-preventive-services-task-force-recommendation. DOI: 10.7326/M15-2886.

TABÁR, L.; YEN, A. M.; WU, W. Y.; CHEN, S. L.; CHIU, S. Y.; FANN, J. C.; KU, M. M.; SMITH, R. A.; DUFFY, S. W.; CHEN, T. H. Insights from the breast cancer screening trials: how screening affects the natural history of breast cancer and implications for evaluating service screening programs. Breast. Journal. v. 21, n. 1, p.13-20, 2015. Disponível em: https://www.ncbi.nlm.nih.gov/pubmed/25413699. DOI:10.1111/tbj.12345. 
TESSER, C. D. Por que é importante a prevenção quaternária na prevenção? Revista de Saúde Pública. v. 51, n. 116, p. 1-9, 2017. Disponível em: http://www.scielo.br/pdf/rsp/v51/pt_0034-8910-rsp-S1518-87872017051000041.pdf. DOI: $10.11606 / \mathrm{S} 1518-8787.2017051000041$.

TOMAZELLI, J. G.; MIGOWSK, A.; RIBEIRO, C. M.; ASSIS, M.; ABREU, D. M. F. Avaliação das ações de detecção precoce do câncer de mama no Brasil por meio de indicadores de processo: estudo descritivo com dados do Sismama, 20102011. Epidemiologia e Serviços de Saúde. v. 26, n.1, p. 61-70, 2017. Disponível em: http://www.scielo.br/scielo.php? script=sci_arttext\&pid=S223796222017000100061\&lng=en\&nrm=iso.

DOI: $0.5123 / \mathrm{s} 1 \overline{6} 79-49742017000100007$.

TORRE, L. A.; BRAY, F.; SIEGEL, R. L.; FERLAY, J.; LORTET-TIEULENT, J.; JEMAL, A. Global cancer statistics, 2012. CA: Cancer Journal for Clinicians. v. 65, n. $2, \quad$ p. 87-108, 2015. Disponível em: https://onlinelibrary.wiley.com/doi/epdf/10.3322/caac.21262. 10.3322/caac.21262.

URBAN, L. A. B. D.; SCHAEFER, M. B.; DUARTE, D. L.; SANTOS, R. P.; MARANHÃO, N. M. A.; KEFALAS, A. L.; CANELLA, E. O.; FERREIRA, C. A. P.; PEIXOTO, J. E.; CHALA, L. F.; COSTA, R. P.; FRANCISCO, J. L. E.; MARTINELLI, S. E.; AMORIM, H. L. E.; PASQUALETTE, H. A.; PEREIRA, P. M. S.; CAMARGO JUNIOR, H. S. A.; SONDERMANN, V. R. Recomendações do Colégio Brasileiro de Radiologia e Diagnóstico por Imagem, da Sociedade Brasileira de Mastologia e da Federação Brasileira das Associações de Ginecologia e Obstetrícia para rastreamento do câncer de mama por métodos de imagem. Radiologia Brasileira. v. $45, \quad$ n. $6, \quad$ p. 334-339, 2012. Disponível em: http://www.scielo.br/pdf/rb/v50n4/pt_0100-3984-rb-50-04-0244.pdf. 10.1590/S0100-39842012000600009.

VASQUEZ, C. L. El Movimiento social del cáncer de mama como dispositivo neoliberal. Revista Estudos Feministas. v. 25, n. 3, p. 1347-1354, 2017. Disponível em: $<$ http://www.scielo.br/scielo.php?script=sci_arttext\&pid=S0104026X2017000301347\&lng=en\&nrm=iso>. DOI: $10.1590 / 1806-$ 9584.2017v25n3p1347. 